\title{
Jmjd2/Kdm4 demethylases are required for expression of Il3ra and survival of acute myeloid leukemia cells
}

\author{
Karl Agger, ${ }^{1,2}$ Satoru Miyagi, ${ }^{1,2,3,4}$ Marianne Terndrup Pedersen, ${ }^{1,2}$ Susanne M. Kooistra, ${ }^{1,2,5}$ \\ Jens Vilstrup Johansen, ${ }^{1}$ and Kristian Helin ${ }^{1,2,3}$ \\ ${ }^{1}$ Biotech Research and Innovation Centre (BRIC), University of Copenhagen, 2200 Copenhagen, Denmark; ${ }^{2}$ Centre for Epigenetics, \\ University of Copenhagen, 2200 Copenhagen, Denmark; ${ }^{3}$ The Danish Stem Cell Center (DanStem), Faculty of Health and Medical \\ Sciences, University of Copenhagen, 2200 Copenhagen, Denmark
}

Acute myeloid leukemias (AMLs) with a rearrangement of the mixed-linage leukemia (MLL) gene are aggressive hematopoietic malignancies. Here, we explored the feasibility of using the H3K9- and H3K36-specific demethylases Jmjd2/Kdm4 as putative drug targets in MLL-AF9 translocated leukemia. Using Jmjd2a, Jmjd2b, and Jmjd2c conditional triple-knockout mice, we show that Jmjd2/Kdm4 activities are required for MLL-AF9 translocated AML in vivo and in vitro. We demonstrate that expression of the interleukin 3 receptor $\alpha$ (Il3ra also known as Cd123) subunit is dependent on Jmjd2/Kdm4 through a mechanism involving removal of H3K9me3 from the promoter of the Il3ra gene. Importantly, ectopic expression of Il3ra in Jmjd2/Kdm4 knockout cells alleviates the requirement of Jmjd2/ $\mathrm{Kdm} 4$ for the survival of AML cells, showing that Il3ra is a critical downstream target of Jmjd2/Kdm4 in leukemia. These results suggest that the JMJD2/KDM4 proteins are promising drug targets for the treatment of AML.

[Keywords: acute myeloid leukemia; histone demethylase; H3K9 methylation; JMJD2; epigenetics; interleukin 3] Supplemental material is available for this article.

Received March 6, 2016; revised version accepted May 4, 2016.

Acute myeloid leukemias (AMLs) with translocations of the mixed-linage leukemia $1(M L L 1)$ gene are aggressive hematopoietic malignancies. MLL1 translocations occur in $\sim 35 \%-50 \%$ of infant AML cases and are frequently found in therapy-related leukemia. Often, MLL1 translocated AML becomes refractory to chemotherapy, and patients have a poor survival compared with patients without MLL1 rearrangements using current treatment protocols. Thus, there is an urgent need for the development of novel therapies (Muntean and Hess 2012; de Boer et al. 2013).

Mechanistic insight into the underlying molecular basis of leukemogenesis driven by MLL1 fusions has expanded significantly within recent years (Cai et al. 2015; Chen and Armstrong 2015). Wild-type MLL1 is a histone methyltransferase with specificity toward $\mathrm{H} 3 \mathrm{~K} 4$ and is required for the transcription of $1.8 \%$ of mammalian genes, including members of the homeobox $(\mathrm{HOX})$ gene cluster (Rao and Dou 2015). H3K4 methyltransferase activity is lost in MLL1 fusions, and the mechanism of leukemogen-

Present addresses: ${ }^{4}$ Department of Cellular and Molecular Medicine, Graduate School of Medicine, Chiba University, Chiba 260-8670, Japan; ${ }^{5}$ Department of Neuroscience, University of Groningen, University Medical Centre Groningen, 9712 Groningen, The Netherlands. Corresponding author: kristian.helin@bric.ku.dk

Article published online ahead of print. Article and publication date are online at http://www.genesdev.org/cgi/doi/10.1101/gad.280495.116. esis is dependent on the fusion partner, most commonly AF4, AF9, and ENL. The current hypothesis is that chimeric MLL1 fusion proteins maintain a leukemia-specific gene expression pattern that is important for the survival of AML cells (Bernt et al. 2011).

Several chromatin-associated enzymes have been found to be required for the growth of MLL-rearranged leukemia, and small molecule inhibitors of some of these enzymes are promising candidates for the development of new drugs (Cai et al. 2015; Chen and Armstrong 2015). The JMJD2 (also known as KDM4) enzymes are histone demethylases with specificity toward $\mathrm{H} 3 \mathrm{~K} 9 \mathrm{me} 3 / \mathrm{me} 2$ and H3K36me3/me2 (Cloos et al. 2006; Fodor et al. 2006; Klose et al. 2006; Whetstine et al. 2006). While H3K9me3 is associated with transcriptional repression, when localized on transcription start sites (TSSs), H3K36me3 is covering actively transcribed regions of chromatin (Kouzarides 2007). The JMJD2/KDM4 protein family consists of four members: JMJD2A, JMJD2B, JMJD2C, and JMJD2D. While the JMJD2A, JMJD2B, and JMJD2C proteins are highly homologous and expressed in most cell types,

(C) 2016 Agger et al. This article is distributed exclusively by Cold Spring Harbor Laboratory Press for the first six months after the full-issue publication date (see http://genesdev.cshlp.org/site/misc/terms.xhtml). After six months, it is available under a Creative Commons License (Attribution-NonCommercial 4.0 International), as described at http://creativecommons.org/licenses/by-nc/4.0/. 
JMJD2D lacks the C-terminal part of the protein and is mainly expressed in testis (Iwamori et al. 2011). The JMJD2/KDM4 enzymes are overexpressed in multiple human cancers, and some studies have shown that they can contribute to tumor cell proliferation (Cloos et al. 2006; Kawazu et al. 2011; Shi et al. 2011; Luo et al. 2012). These observations, in combination with a well-defined catalytic mechanism, define these enzymes as attractive drug targets (Kooistra and Helin 2012; Berry and Janknecht 2013; Hojfeldt et al. 2013).

In this study, we used mice in which $\operatorname{Imj} d 2 a$, Jmjd2b, and $J m j d 2 c$ are conditionally deleted to probe the therapeutic potential of targeting Jmjd2/Kdm4 activity in a mouse model of MLL-AF9-driven leukemia.

\section{Results}

Imjd2a, Jmjd2b, and Jmjd2c are required for progression of MLL-AF9 translocated leukemia in vivo

Retroviral-mediated expression of MLL-AF9 can transform general myeloid progenitors (GMPs) into immortalized leukemic blast cells in vitro, and mice transplanted with these cells develop AML (Krivtsov et al. 2006; Somervaille and Cleary 2006). Using this system in combination with knockout mouse strains that we generated (Pedersen et al. 2016), we developed a mouse model of MLL-AF9 translocated AML that is conditionally knocked out for Jmjd2/Kdm 4 activity. In cells from these mice, loxP sites are flanking critical exons in Imjd2a, $J m j d 2 b$, and $J m j d 2 c$, and, in addition, an inducible form of Cre recombinase (CreERT2) is expressed from the Rosa26 locus (we refer to this mouse strain as $2 a b c$; CreER). The activity of CreERT2 can be activated by 4-hydroxytamoxifen $(\mathrm{OHT})$ in vitro and in vivo.
We isolated $\mathrm{c}-\mathrm{Kit}^{+}$cells from the bone marrow (BM) of Jmjd2c;CreER and Imjd2abc;CreER mice. The cells were transduced with a retrovirus expressing MLL-AF9, plated in methocult medium, and subsequently serially replated three times to enrich for preleukemic GMPs (denoted as pre-MA9-2c and pre-MA9-2abc) (Fig. 1A).

To investigate the role of Jmjd2/Kdm4 in MLL-AF9-induced transformation of GMPs, we examined the cell growth and colony-forming capability of preleukemic GMPs. Addition of OHT to the growth medium resulted in efficient depletion of $I m d 2 a$, Imjd2b, and Imjd2c in preMA9-2abc and Imjd2c in pre-MA9-2c cells (Supplemental Fig. 1A). Strikingly, the deletion of $\operatorname{Imj} d 2 a$, $\operatorname{Imj} d 2 b$, and $\operatorname{Imjd2c}$, but not $I m j d 2 c$ alone, led to a strong attenuation of growth in liquid culture (Supplemental Fig. 1B).

Having established that the combined activity of Jmjd2a, Jmjd2b, and Jmjd2c is required for the growth of preleukemic GMPs, we tested whether these proteins also are required for MLL-AF9 translocated leukemia in vivo. We transplanted pre-MA9-2abc and pre-MA9-2c cells into sublethally irradiated recipient mice (Fig. 1A). At day 21 after transplantation, the mice were injected with tamoxifen (converted to OHT in the mouse liver) daily over a period of 10 consecutive days to induce knockout of Imjd2a, Imjd2b, and Imjd2c. While deletion of Imjd2c alone did not have any significant effect on mouse (leukemic) survival (Fig. 1B), the combined deletion of $J m j d 2 a$, Imjd2b, and $J m j d 2 c$ resulted in a substantial extension of life span of the mice $(P=0.0009)$ (Fig. 1C). In these experiments, FACS analyses of spleen cells from leukemic mice were performed to confirm the emergence of AML; i.e., infiltration of $\mathrm{Gr}^{+} / \mathrm{Mac1}^{+}$-positive cells in the spleen (Fig. 1D). Two tamoxifen-treated MA9-2abc mice became leukemic (Fig. 1C), but, notably, genotyping showed that the leukemic cells from one of these mice
A

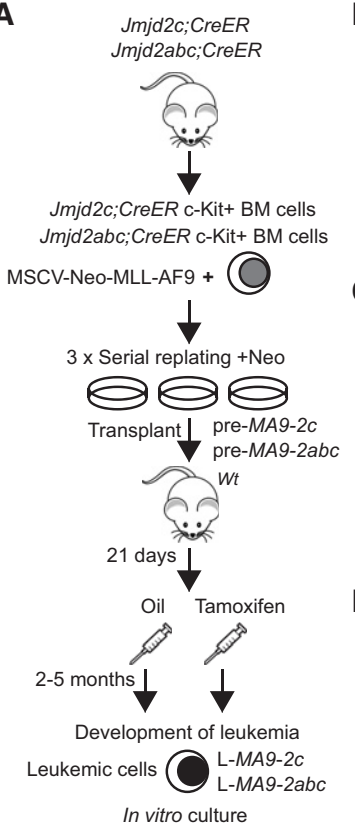

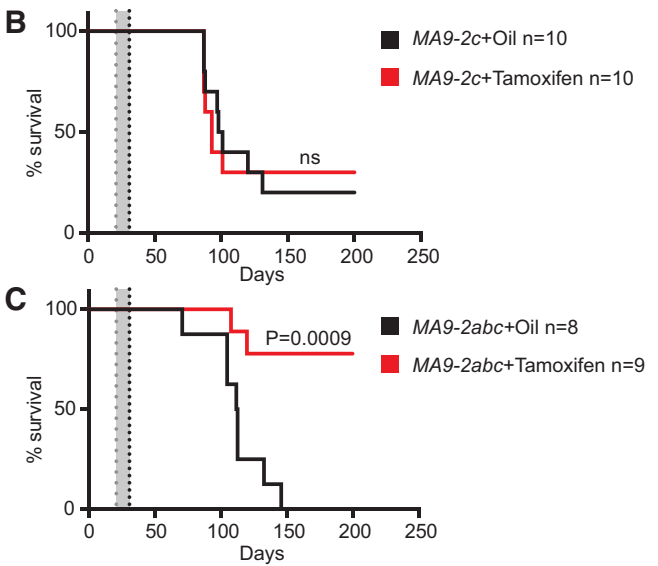

D

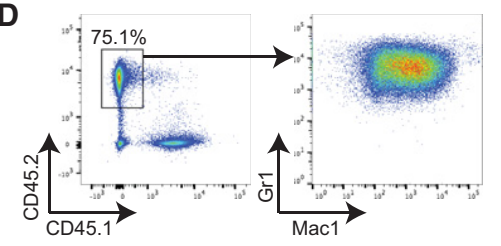

Figure 1. MLL-AF9 cells are dependent on the combined activity of Jmjd2a, Jmjd2c, and Jmjd2c in vivo. (A) Schematic drawing of the experimental setup. $(B, C)$ Kaplan-Meier curve depicting the survival of mice transplanted with $5 \times 10^{4}$ preleukemic cells with the indicated genotypes. Twenty-one days after transplantation, the mice were injected daily with $1 \mathrm{mg}$ of tamoxifen dissolved in oil or with oil alone for a period of $10 \mathrm{~d}$ (indicated as a gray area in the growth curve). The median survival of MA9-2abc mice injected with oil was $112.5 \mathrm{~d}$; for MA9-2abc mice injected with tamoxifen, the median survival was not reached; for MA9-2c mice injected with oil, the median survival was $99.5 \mathrm{~d}$; and for MA9-2c mice injected with tamoxifen, the median survival was 93 d. $P$-values are the result of a Mantel-Cox statistical test. $(D)$ Representative FACS plots of cells from the spleen of a leukemic mouse. Plots show $\mathrm{Grl}^{+}$and $\mathrm{Macl}^{+}$double-positive in the CD45.2 gate. 
had retained the wild-type alleles and therefore represented an escaper clone (data not shown).

To investigate the effect of deleting $\operatorname{Imj} d 2 a$, $\operatorname{Imj} \mathrm{I} 2 \mathrm{~b}$, and $I m j d 2 c$ on normal hematopoietic development, we reconstituted the hematopoietic system in lethally irradiated mice using untransformed BM cells from 2abc; CreER mice or wild-type controls. Injection of tamoxifen into these mice resulted in efficient recombination of the floxed alleles of $J m j d 2 a$, $\operatorname{Imj} d 2 b$, and $J m j d 2 c$ in CD45.2positive donor cells, as evident upon FACS sorting of peripheral blood (Supplemental Fig. 2A). However, we did not observe any significant changes in overall CD45.2 chimerism or survival of the mice within a 3-mo period (Supplemental Fig. 2B,C). These results demonstrate that hematopoiesis can develop to some extent in the absence of $\operatorname{Jmjd} 2 \mathrm{a}$, Jmjd2b, and Jmjd2c.

Taken together, we conclude that the simultaneous knockout of $\operatorname{Imj} d 2 a, \operatorname{Imj} d 2 b$, and $J m j d 2 c$ perturbs the progression of MLL-AF9 translocated leukemia in mice, whereas inactivation of $I m j d 2 c$ alone does not have any effect. Likewise, we found that knockout of Imjd2a, $I m j d 2 b$, and $I m j d 2 c$ does not result in any severe phenotype in untransformed BM cells and that Imjd2abc;CreER donor cells can contribute sufficiently to the hematopoietic system to make recipients survive.

Loss of Imjd2/Kdm4 compromises the proliferative capacity of MLL-AF9 transformed GMPS (L-GMPS)

Having established that Jmjd2/Kdm4 is required for $\mathrm{AML}$ in vivo, we wanted to understand how loss of Jmjd2/Kdm 4 affects the growth of L-GMPs. To do this, we examined the growth and colony formation capability of leukemic cells isolated from the BM of moribund pre-MA9-2c and pre-MA9-2abc transplanted mice that had been injected with oil. In these cells, $\operatorname{Imj} d 2 a, \operatorname{Imj} d 2 b$, and $J m j d 2 c$ are still wild type, and genetic inactivation of the $I m j d 2 /$ $\mathrm{Kdm} 4$ genes can be induced in vitro. Treatment of these cells in liquid culture for $96 \mathrm{~h}$ with $500 \mathrm{nM}$ OHT resulted in an efficient depletion of $\operatorname{Jmj} 2 \mathrm{C}$, Jmjd2b, and $\operatorname{Jmjd} 2 \mathrm{c}$ protein and mRNA (Fig. 2A,B).

We found that proliferation of two independently isolated leukemic MA9-2abc cell lines (L-MA9-2abc-1 and L-MA9-2abc-2) in liquid culture was severely compromised upon OHT treatment, whereas the proliferation of L-MA9-2c cells was unaffected (Fig. 2C). Similarly, the ability to form colonies in methylcellulose was lower for L-MA9-2abc cells treated with OHT compared with untreated cells, whereas no effect of OHT treatment was observed for L-MA9-2c cells (Fig. 2D). Deletion of Imjd2a, $I m j d 2 b$, and Imjd2c resulted in increased levels of cell death, elevated levels of Mac1, and slightly reduced numbers of S-phase cells (Fig. 2E-G) as well as an increased number of differentiated cells (Fig. 2H). In contrast, we observed only a modest effect on growth of untransformed c-Kit-positive BM cells upon knockout of $\operatorname{Imj} \mathrm{d}_{2} a$, Jmjd2b, and $\operatorname{mid2c}$ (Fig. 2I), which is in agreement with our in vivo observations (Supplemental Fig. 2A-C).

In summary, these data suggest that the extended life span of leukemic mice after genetic inactivation of $\operatorname{Imj} d 2 a$,
$I m j d 2 b$, and $I m j d 2 c$ is the result of enhanced levels of differentiation and cell death of the leukemic cells.

Imjd2/Kdm4 proteins are required for the survival of $M L L$-AF9 transformed cells independently of Hoxa9 and Meis 1

MLL-AF9 directly regulates the transcription of Hoxa9 and Meis1, which are essential downstream targets for MLL-AF9 during leukemic transformation. This is underscored by the fact that coexpression of Hoxa9 and Meis1 is sufficient to induce transformation of GMPs (Kroon et al. 1998; Wang et al. 2010). In a recent study published while this work was in preparation, it was reported that Jmjd2c binds to MLL-AF9 and is an essential cofactor for the transcriptional activation of Hoxa9 and Meis1 during leukemic transformation (Cheung et al. 2016). According to this study, Jmjd2c is recruited to the TSSs of Hoxa9 and Meis1, where it can demethylate H3K9me3 (Cheung et al. 2016). Additionally, it was reported that Jmjd2c alone is required for leukemic transformation by MLLAF9 (Cheung et al. 2016), a conclusion that contrasts with our data showing that deletion of $J m j d 2 c$ alone has no effect on leukemic transformation, while only the simultaneous deletion of $\operatorname{Imj} j 2 a, \operatorname{Imj} d 2 b$, and $\operatorname{Imjd2c}$ results in attenuation of leukemic growth. To understand whether transcription of Hoxa9 and Meis1 was affected upon genetic inactivation of $I m j d 2 a$, Imjd2b, and $I m j d 2 c$, we performed RT-qPCR analysis on L-MA9-2c and L-MA9-2abc cells. However, we did not observe any significant effect on Hoxa9 and Meis1 mRNA levels upon OHT-induced deletion of $I m j d 2 c$ alone or from the simultaneous deletion of $\operatorname{Imj} d 2 a, \operatorname{Imj} d 2 b$, and $J m j d 2 c$ (Fig. 3A).

To further address whether Hoxa9 and Meis1 could have a role in the observed growth defect, we took advantage of the fact that GMPs can be transformed by the coexpression of Hoxa9 and Meis1 (Kroon et al. 1998). We speculated that if $\mathrm{Jmjd} 2 / \mathrm{Kdm} 4$, similar to Dot1L (Bernt and Armstrong 2011), is genetically upstream of Hoxa9 and Meis1, then cells transformed with Hoxa9 and Meis1 would be unresponsive to knockout of $/ \mathrm{mid} / \mathrm{Kdm} 4$. On the other hand, if the growth defect is still observed in Hoxa9-Meis1 transformed cells, then the Jmjd2/Kdm 4 proteins are required downstream from Hoxa9 and Meis1 or in a parallel independent pathway. We purified c-Kit ${ }^{+}$ cells from the BM of Imjd2c;CreER and Imjd2abc;CreER mice and transduced them with retroviruses coexpressing Hoxa9 and Meis1. After three rounds of replating in methylcellulose, the cells were transferred to liquid medium and treated with $500 \mathrm{nM}$ OHT for $96 \mathrm{~h}$. As observed in MLL-AF9 transformed cells, OHT treatment resulted in efficient knockout of $\operatorname{Imj} d 2 a, \operatorname{Imj} d 2 b$, and Imid2c (Fig. 3B). This led to an even stronger effect on growth than that observed in MLL-AF9 transformed cells, whereas knockout of $\operatorname{Imj} d 2 \mathrm{c}$ alone did not have any effect (Fig. 3C).

From these data, we conclude that Jmjd2a, Jmjd2b, and $\mathrm{Jmjd} 2 \mathrm{c}$ are required for maintaining the transformed phenotype of MLL-AF9 leukemia downstream from Hoxa9 and Meis1 or in a parallel independent pathway. 
A

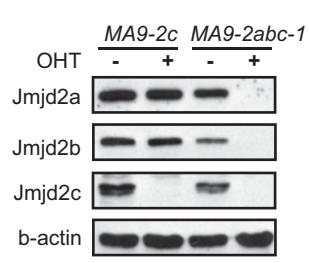

B

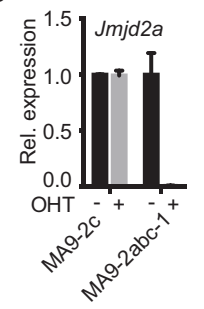

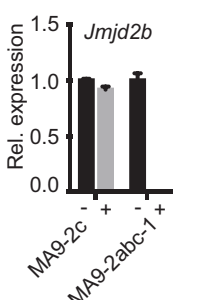

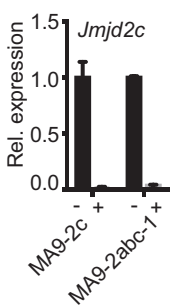

C

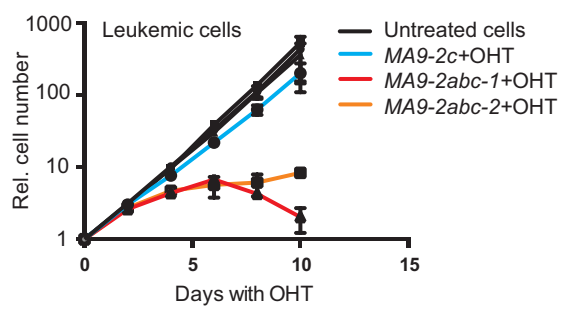

D

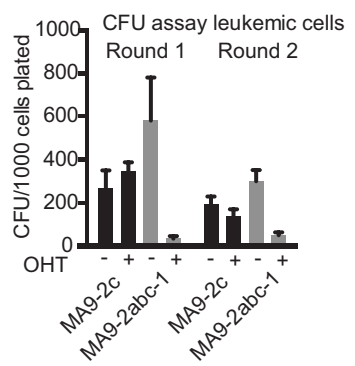

$\mathbf{E}$

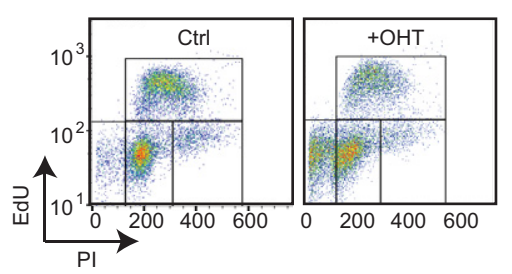

H

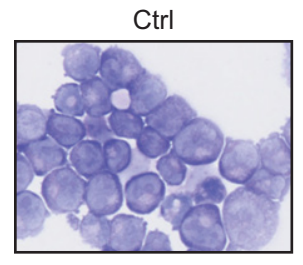

F

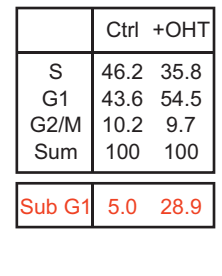

G

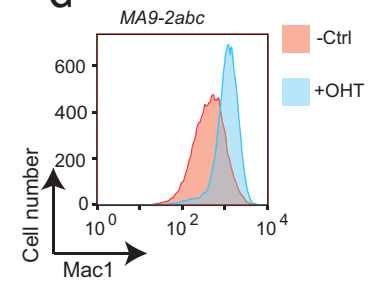

I
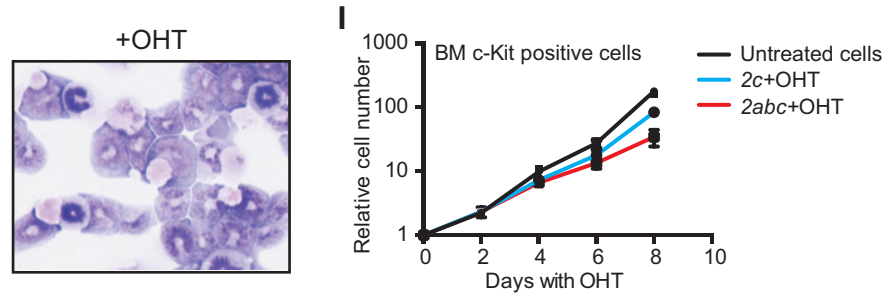

Figure 2. Loss of Jmjd2/Kdm4 activity results in decreased growth combined with an increase in differentiation and cell death for MLLAF9 transformed leukemic cells. (A) Western blot documenting efficient depletion of Jmjd2a, Jmjd2b, and Jmjd2c protein levels in MLL-AF9 transformed murine leukemic cells after $96 \mathrm{~h}$ of treatment with $500 \mathrm{nM} \mathrm{OHT}$. (B) RT-qPCR from the indicated cells, confirming efficient depletion of full-length $I m j d 2 a, J m j d 2 b$, and $J m j d 2 c$ mRNA after OHT treatment. (C) Growth curve of three different leukemic cell lines (LMA9-2c, L-MA9-2abc-1, and L-MA9-2abc-2) in liquid culture with or without $500 \mathrm{nM}$ OHT. The cell lines were established from leukemic mice transplanted with preleukemic cells of the indicated genotypes. (D) Blast colony formation assay of MA9-2c and MA9-2abc leukemic cells in methylcellulose. Cells were plated after $4 \mathrm{~d}$ of treatment with $500 \mathrm{nM}$ OHT in liquid medium. (E) FACS analysis of EdU incorporation in MA9-2abc cells showing an increased sub-G1 fraction, indicative of cell death. $(F)$ The top panel represents cell cycle distribution of MA9-2abc cells, excluding the sub-G1 fraction. The bottom red panel shows the percentage of cells with a sub-G1 (<2N) DNA content. The cells were treated for $96 \mathrm{~h}$ with $500 \mathrm{nM}$ OHT prior to the analysis. (G) FACS plot showing increased Mac1 staining in L-MA9-2abc cells treated with OHT for $96 \mathrm{~h}$ prior to the analysis. $(H)$ May-Grünwald-Giemsa staining of L-MA9-2abc cells treated with OHT for $96 \mathrm{~h}$. $(I)$ Growth curve of c-kit ${ }^{+}$-enriched untransformed progenitors from 2abc;CreER and 2c;CreER in liquid culture with or without treatment of OHT. The graphs show mean \pm SD of technical replicates and are representative of at least three independent experiments.

\section{Imjd2 a and Imjd2c bind to H3K4me3-positive TSSs in MLL-AF9 transformed cells}

Having established that the requirement of the Jmjd2/ Kdm4 proteins for the survival of MLL-AF9 transformed AML cells cannot be explained by the lack of Hoxa9 and Meis1 expression, we performed unbiased genome-wide studies (i.e., genome-wide location analysis in combination with expression studies). We mapped the binding patterns of Jmjd2a and Jmjd2c in L-MA9-2abc cells by chromatin immunoprecipitation (ChIP) combined with high-throughput sequencing (ChIP-seq). Due to the lack of ChIP-grade antibodies, a similar analysis was not performed for Jmjd2b (data not shown). Using OHT-treated L-MA9-2abc cells devoid of Jmjd2 proteins as a negative control, bioinformatics analysis identified 8977 significantly bound regions representative of Jmjd2a-binding sites, of which $77 \%$ were localized within $\pm 1 \mathrm{~kb}$ of an annotated TSS. For Jmjd2c, we identified 10,521 significantly bound regions, of which $86 \%$ localized within 
A

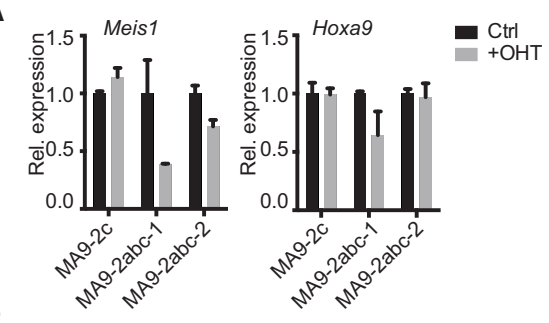

B

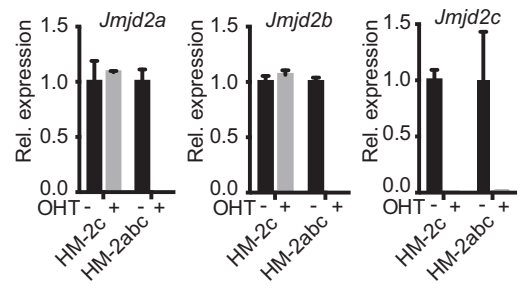

C

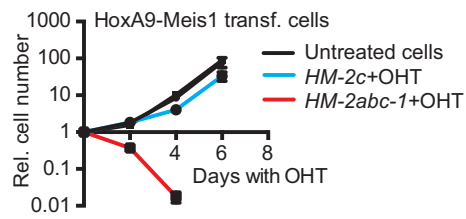

Figure 3. Jmjd2/Kdm4 proteins are required for the survival of MLL-AF9 transformed cells downstream from Hoxa9 and Meis1. (A) RT-qPCR of Hoxa9 and Meis1 using mRNA prepared from two independently derived L-MA9-2abc cell lines and one L-MA9-2c cell line treated with or without $500 \mathrm{nM}$ OHT for 96 h. (B) Depletion efficiency of $I m j d 2 a, I m j d 2 b$, and $I m j d 2 c$ measured by RT-qPCR in HoxA9-Meis1 (HM) transformed preleukemic cells after $96 \mathrm{~h}$ of treatment with $500 \mathrm{nM}$ OHT. $(C)$ Growth curve of the same cells as in $B$ in liquid culture. The growth curve was started after $96 \mathrm{~h}$ of OHT treatment. The graphs show mean \pm SD of technical replicates and are representative of at least three independent experiments.

$\pm 1 \mathrm{~kb}$ of a TSS (Fig. 4A). The binding of Jmjd2a and Jmjd2c was confirmed in an independent biological experiment by ChIP-qPCR on a selected target (Fig. 4B). Although the phenotype that we observed upon Jmjd2/Kdm 4 depletion was not dependent on deregulated Hoxa9 and Meis1 transcription, Jmjd2c was found to bind to the promoter of Meis1 and Hoxa9, in agreement with Cheung et al. (2016) (Supplemental Fig. 3A,B).

Previously, we reported that Jmjd2a and Jmjd2c both localize to H3K4me3-positive TSSs in mouse embryonic stem cells (mESCs) (Pedersen et al. 2014, 2016). Similar to what was observed for Jmjd2a and Jmjd2c in mESCs, we found a strong overlap in Jmjd2a- and Jmjd2c-binding patterns (Fig. 4C,D; Supplemental Fig. 4A,B) and observed that the vast majority of Jmjd2a- and Jmjd2c-binding sites overlap H3K4me3-marked regions (Fig. 4D; Supplemental Fig. 4C,D). In this way, we found that $94 \%$ and $97 \%$ of TSS-associated Jmjd2a and Jmjd2c peaks directly overlap an H3K4me3 peak (Supplemental Fig. 4C,D) and that 6940 TSS regions are bound by both Jmjd2a and Jmjd2c in L-MA9-2abc cells (Fig. 4C). This is in agreement with a model in which Jmjd2a and Jmjd2c exert redundant functions at H3K4me3-marked TSSs in L-MA92 abc cells.
The Jmjd2/Kdm4 enzymes catalyze demethylation of Lys9 and Lys36 on histone H3. To identify regions that change H3K9me3 and H3K36me3 levels after genetic inactivation of $\operatorname{Imj} d 2 a, \operatorname{Imj} d 2 b$, and $\operatorname{Imj} d 2 c$, we performed ChIP-seq experiments. Here, we used antibodies specifically recognizing $\mathrm{H} 3 \mathrm{~K} 9 \mathrm{me} 3$ and $\mathrm{H} 3 \mathrm{~K} 36 \mathrm{me} 3$ as well as chromatin from control L-MA9-2abc cells or L-MA92 abc cells that had been treated with OHT for $96 \mathrm{~h}$. The heat maps (Fig. 4E; Supplemental Fig. 5A) present two biologically independent experiments and depict the H3K9me3 and H3K36me3 reads around the 6940 TSSs cobound by Jmjd2a and Jmjd2c in L-MA9-2abc cells cultured in the absence or presence of OHT. It is evident that the H3K9me3 levels are strongly increased by the simultaneous deletion of $\operatorname{Imj} j 2 a, \operatorname{Imj} d 2 b$, and $\operatorname{Imj} d 2 c$ on a subset of the cobound TSSs (Fig. 4E, top part of the heat map). Increased $\mathrm{H} 3 \mathrm{~K} 9 \mathrm{me} 3$ levels were confirmed by ChIP-qPCR on selected Jmjd2a and Jmjd2c target genes (Fig. 4B). For H3K36me3, we also observed an increase in methylation levels, shifting the profiles toward the TSS at $\operatorname{Jmj} \mathrm{d} 2 \mathrm{a} / \mathrm{Jmj} \mathrm{d} 2 \mathrm{c}$-cobound regions upon $\mathrm{Jmj} \mathrm{d} 2 / \mathrm{Kdm} 4$ depletion (Supplemental Fig. 5A).

Taken together, we demonstrated that Jmjd2a and Jmjd2c both localize to H3K4me3-positive TSSs in MLLAF9 transformed leukemic cells, where they prevent accumulation of $\mathrm{H} 3 \mathrm{~K} 9 \mathrm{me} 3$ and $\mathrm{H} 3 \mathrm{~K} 36 \mathrm{me} 3$.

\section{Imid2-mediated H3K9me3 demethylation is required for the expression of interleukin 3 receptor $\alpha$ (I13ra)}

We hypothesized that Jmjd2/Kdm4 proteins are required for the transcription of a gene or group of genes in L-MA9-2abc cells and that loss of expression of some of these genes could explain the observed growth defect. To identify such genes, we performed gene expression analysis on three independently derived L-MA9-2abc cell lines cultured in the absence or presence of OHT for 96 $\mathrm{h}$. When setting the cutoff at an absolute fold change of $>2$ with a false discovery rate (FDR) of $<0.05$, we found that the combined loss of $I m j d 2 a, I m j d 2 b$, and $I m j d 2 c$ led to down-regulation of 94 genes and up-regulation of 55 genes (Fig. 5A). Thirty of the down-regulated genes (32\%) and 11 of the up-regulated genes $(20 \%)$ contained binding sites for both Jmjd2a and Jmd2c within $\pm 1 \mathrm{~kb}$ of a TSS (Fig. 5B). Gene set enrichment analysis (GSEA) of the array data did not reveal any enrichment of HoxA9/Meis1 target genes, indicating that $\mathrm{Jmj} \mathrm{d} 2 / \mathrm{Kdm} 4$ proteins are not functioning directly downstream from HoxA9/Meis1 but rather in a parallel independent pathway (data not shown).

Loss of Jmjd2/Kdm 4 can result in both direct and indirect effects on transcription; however, the direct targets of $\mathrm{Jmjd} 2 / \mathrm{Kdm} 4$ are expected to have increased levels of H3K9me3 or H3K36me3 at their TSSs upon depletion of Jmjd2 proteins. In order to link transcription and histone methylation, we analyzed H3K9me3 and H3K36me3 levels at TSSs of deregulated genes. Similar to our observations in mESCs, we found a correlation between increased $\mathrm{H} 3 \mathrm{~K} 9 \mathrm{me} 3$ levels and transcriptional repression of Jmjd2a/ c targets, whereas the data did not reveal a link between Jmjd2/Kdm4-dependent control of H3K36me3 
A
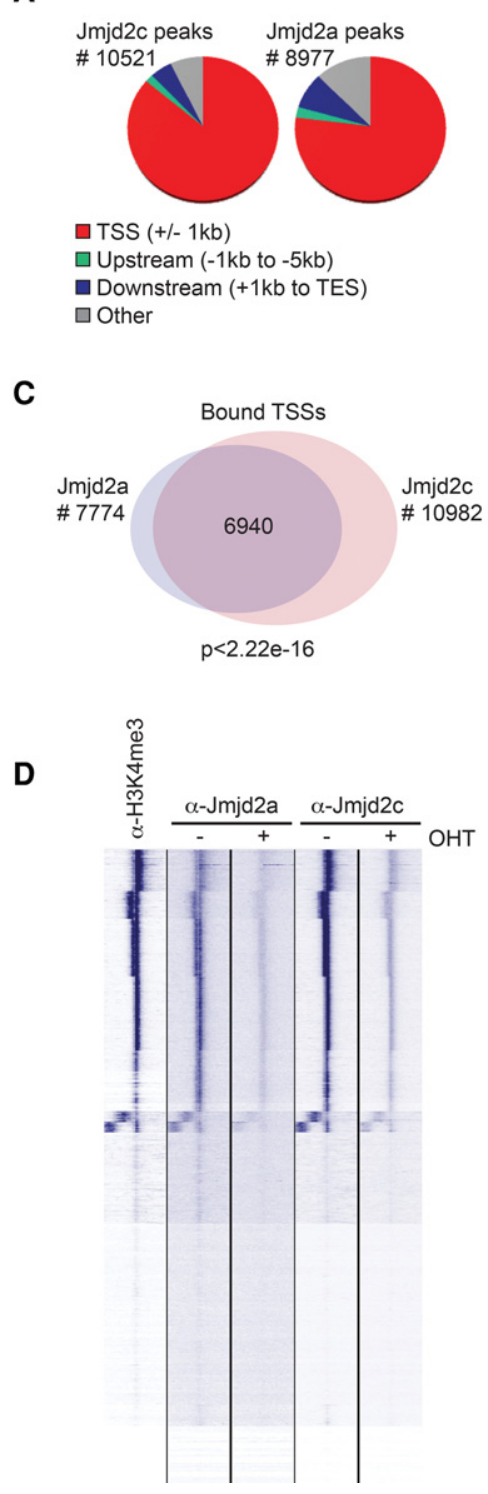

B
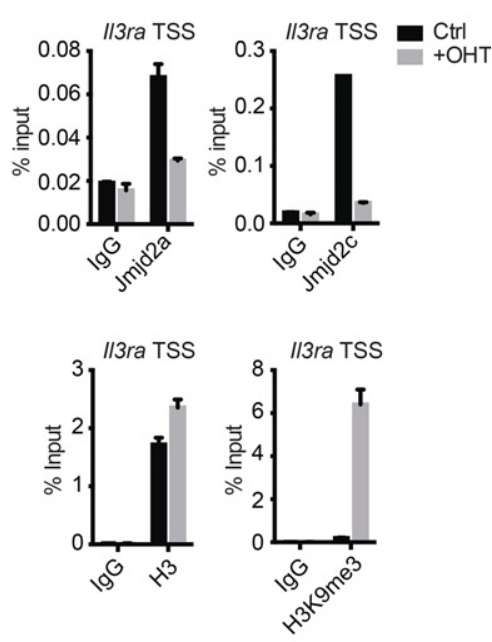

E

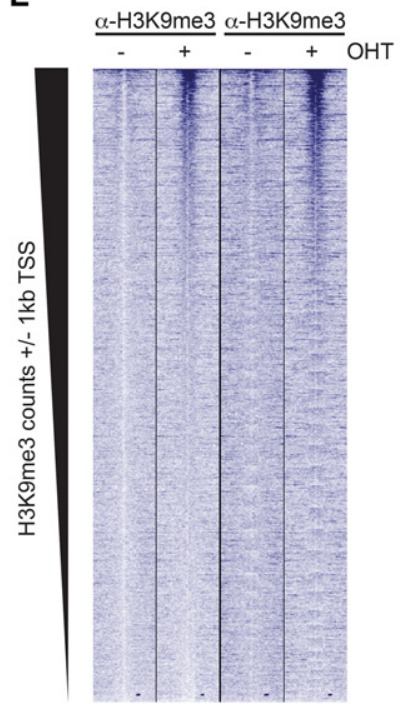

Figure 4. ChIP-seq analyses of Jmjd2a, Jmjd2c, and H3K9me3 in MA9-2abc. (A) Pie diagrams showing the distribution of Jmjd2a- and Jmjd2c-binding sites relative to annotated TSSs and transcription end sites (TESs) in L-MA9-2abc cells. (B) Independent ChIPqPCR validation of ChIP-seq data on a selected TSS cobound by Jmjd2a and Jmjd2c, showing increased H3K9me3 levels after OHT treatment. (C) Venn diagram showing the number of TSSs containing binding sites for Jmjd2a and/or Jmjd2c within $\pm 1 \mathrm{~kb}$. The $P$-value was calculated using a hypergeometric test. $(D)$ Unsupervised $k$-means clustering of ChIP-seq tags over all TSSs $( \pm 5 \mathrm{~kb})$. H3K4me3 ChIP-seq data were from Bernt et al. (2011). (E) H3K9me3 ChIP-seq tags over Jmjd2a/c cobound TSSs $\pm 5 \mathrm{~kb}$ as defined in $C$, ranked by read counts in the +OHT condition. The graphs show mean \pm SD of technical replicates and are representative of at least three independent experiments. and transcriptional changes (Supplemental Fig. 5B,C; Pedersen et al. 2016).

Among the 30 down-regulated genes cobound by Jmjd2a and Jmjd2c, we identified five genes that showed a more than twofold increase in $\mathrm{H} 3 \mathrm{~K} 9 \mathrm{me} 3$ levels upon loss of Jmjd2/Kdm4 expression in both ChIP-seq experiments (Fig. 5A). Among these five genes, we reasoned that Il3ra (also known as Cd123) could be a strong candidate for mediating the phenotype. Il3ra is the a subunit of the heterodimeric Il-3 receptor that, together with the $\beta$ subunit (Il3 receptor $\beta$ ), forms a functional high-affinity receptor. Binding of Il-3 to the receptor results in activation of receptorassociated Janus kinases (JAKs) and its downstream STAT pathway, which ultimately leads to stimulation of proliferation. A considerable number of results have indicated that IL-3 and its receptor play important roles in the survival of AML as well as in inflammation (Broughton et al. 2012; Testa et al. 2014).
We validated the transcriptional down-regulation of II3ra by RT-qPCR in two different L-MA9-2abc cell lines (Fig. 5C). In contrast, we observed only a minor decrease in expression of Il3ra upon OHT treatment of the L-MA9-2c control cell line (Fig. 5C). Moreover, we confirmed that Jmjd2a and Jmjd2c colocalize with H3K4me3 at the TSS of II3ra and that genetic deletion of Imjd2a, Imjd2b, and $J m j d 2 c$ led to an increase in H3K9me3 levels at the TSS (Figs. 4B, 5D). These data strongly suggest that the $\mathrm{Jmjd} 2 / \mathrm{Kdm} 4$ proteins are required for the expression of Il3ra through a process involving the specific demethylation of H3K9 at the TSS of the gene. In agreement with this, GSEA revealed impaired expression of genes downstream from Il3ra in cells depleted for Jmjd2a, Jmjd2b, and Jmjd2c (Fig. 5E; Supplemental Fig. 3E).

To investigate whether Il3ra is a critical downstream target of the Jmjd2/Kdm4 proteins in L-MA9-2abc cells, we performed a genetic rescue experiment. We cloned 
Agger et al.

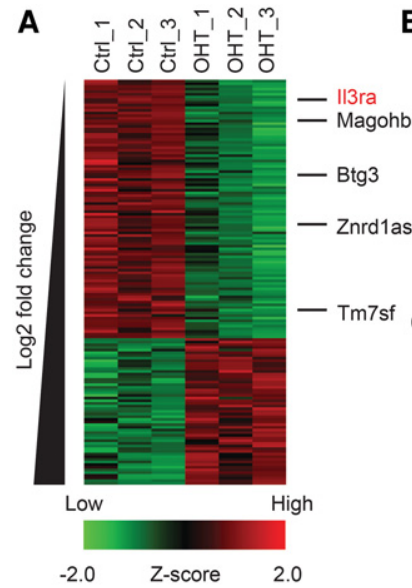

E
B 100 "Not bound

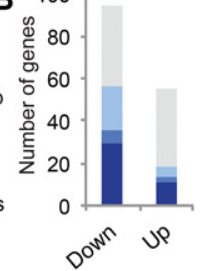

"Bound $2 \mathrm{c}$ only

- Bound 2a only

- Bound $2 \mathrm{a}$ and $2 \mathrm{c}$

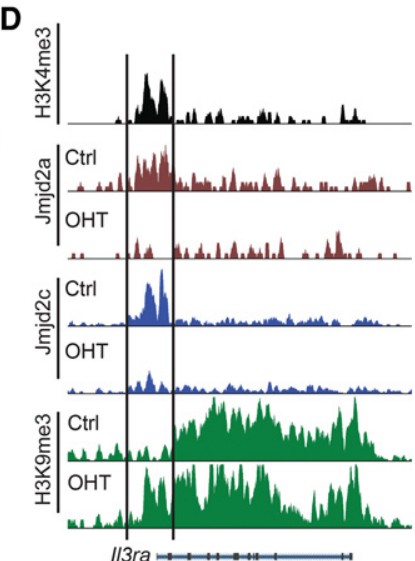

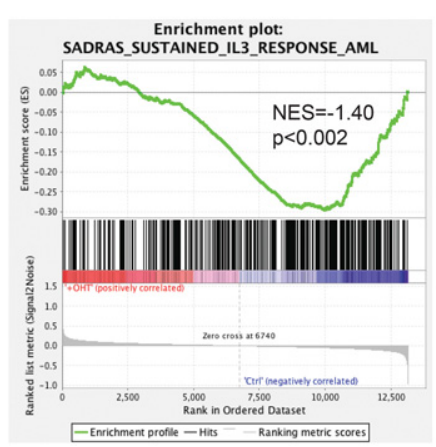

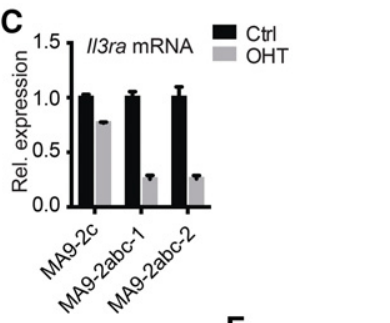

$\mathbf{F}$

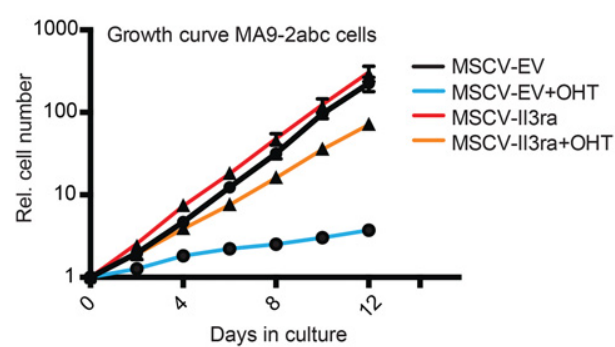

Figure 5. Jmjd2/Kdm4 activity is required for the expression of Il3ra in MA9-2abc cells. (A) Heat map representing gene expression analysis of L-MA9-2abc cells after $96 \mathrm{~h}$ of treatment with $500 \mathrm{nM}$ OHT. Data are presented for genes with deregulated expression (fold change $>2 ;$ FDR $<0.05$ ), genes cobound by Jmjd2a and Jmjd2c and displaying a more than twofold increase in H3K9me3 levels are indicated. (B) Bar diagram showing the number of deregulated genes (fold change $>2$; FDR $<0.05$ ) containing binding sites for Jmjd2a and/or Jmjd2c within \pm 1 $\mathrm{kb}$ of a TSS. (C) RT-qPCR validation of transcriptional repression of Il3ra in two independently derived L-MA9-2abc cell lines and one L-MA9-2c cell line. (D) H3K4me3 (Bernt et al. 2011), Jmjd2a, Jmjd2c, and H3K9me3 ChIP-seq tracks of the Il3ra locus with and without combined depletion of Jmjd2a, Jmjd2b, and Jmjd2c. The area around the TSS is indicated by two vertical lines. (E) GSEA plot showing impaired expression of a gene set induced by IL-3 treatment in human AML samples (Sadras et al. 2014) in OHT-treated L-MA9-2abc cells. (NES) Normalized enrichment score; (p) nominal $P$-value. $(F)$ Growth curve of MA9-2abc cells transduced with MSCV-Il3ra or empty MSCV vector. Cells were grown in the absence or presence of $500 \mathrm{nM} \mathrm{OHT} \mathrm{in} \mathrm{liquid} \mathrm{culture.} \mathrm{Data} \mathrm{are} \mathrm{presented} \mathrm{as} \mathrm{mean} \pm$ SD of technical replicates and are representative of at least three independent experiments.

the ORF of the murine Il3ra cDNA into a retroviral expression vector containing a selectable marker (MSCVIl3ra) and transduced L-MA9-2abc cells with this construct. As shown in Figure 5F, ectopic expression of Il3ra in L-MA9-2abc cells resulted in a rescue of the growth defect observed upon Jmjd2/Kdm4 depletion by OHT treatment.

In conclusion, we found that $\mathrm{Jmj} \mathrm{d} 2 / \mathrm{Kdm} 4$ proteins are required for the leukemic growth of AMLs driven by the MLL-AF9 fusion protein. Specifically, the Jmjd2/Kdm4 proteins are required for the expression of II3ra, and ectopic expression of Il3ra alleviates the requirement for Jmjd2/ $\mathrm{Kdm} 4$ proteins in MLL-AF9 translocated AMLs.

\section{Discussion}

The results presented here demonstrate that targeting of Jmjd2/Kdm4 activity in MLL-AF9 translocated AML is a feasible strategy for the development of novel therapies.
We show that depletion of Jmjd2/Kdm4 activity through the simultaneous genetic inactivation of $\operatorname{Imj} d 2 a$, Imjd2b, and $I m j d 2 c$ is deleterious to the survival of leukemic cells in a mouse model of MLL-AF9 translocated AML, whereas depletion of Jmjd2c alone does not have any effect, indicating redundant roles of Jmjd2a, Jmjd2b, and Jmjd2c in AML. We also show that Jmjd2/Kdm4 depletion in nontransformed c-Kit ${ }^{+} \mathrm{BM}$ cells in vitro as well as in vivo has a less pronounced effect on cellular growth, opening a therapeutic window of opportunity for the targeting of Jmjd2/Kdm4 activity in AML.

A report published while this study was in preparation suggests an essential role for Jmjd2c alone in the survival MLL-AF9 translocated AML (Cheung et al. 2016). However, our results differ from the published observations in two conceptually important ways. First, we did not see any effect on the survival of MLL-AF9 translocated AML upon deletion of $J m j d 2 c$ alone either in vivo or in vitro. Second, our data do not support a model in which Jmjd2c 
is required for the regulation of Hoxa9 and Meis1 transcription. Although these differences can be hard to reconcile, the first discrepancy could be due to the fact that experiments presented by Cheung et al. (2016) mainly relied on the usage of shRNA, which can have substantial off-target effects. In contrast, in our studies, we used a genetic knockout model that is not associated with offtarget effects. We did not test whether the Jmjd2/Kdm 4 proteins can bind to MLL-AF9; however, our results do not support a model in which Hoxa9 and Meis1 are critical targets for the Jmjd2/Kdm 4 proteins in AML because we did not observe a major effect on Hoxa9 and Meis1 expression in the absence of the Jmjd2/Kdm4 proteins. Moreover, our genetic studies show that the Jmjd2/Kdm 4 proteins are required downstream from Hoxa9 and Meis1 or in a parallel independent pathway.

To identify the direct targets for Jmd2/Kdm 4 that could explain the requirements for these proteins in MLL-AF9 translocated AMLs, we mapped the genomic binding sites for Jmjd2a and Jmjd2c in mouse leukemic cells. Using our triple-knockout cells, we identified functionally important binding sites where loss of Jmjd2/Kdm4 was coincidental with increased H3K9me3 levels. The combination of genome-wide mapping analysis and gene expression analysis enabled us to pinpoint II3ra as an essential transcriptional target of $\mathrm{Jmjd} 2 / \mathrm{Kdm} 4$ proteins in AML.

I13ra (Cd123) is a nonessential gene in mice (Nishinakamura et al. 1995). It has previously been found overexpressed in several types of leukemia, such as AML, B-progenitor acute lymphoblastic leukemia (B-ALL), T-cell ALL (T-ALL), and chronic lymphocytic leukemia (CLL), and targeting IL3RA-expressing cells using neutralizing antibodies against IL3RA as well as IL3RA antibodies coupled to toxins has shown beneficial effects on survival in AML models (Jordan et al. 2000; Testa et al. 2002; Jin et al. 2009; Liu et al. 2015). Notably, two phase I clinical trials using IL3RA monoclonal antibodies for the treatment of AML are currently being completed (http://www.clinicaltrials.gov); the results from these trials have not been published yet, and the outcomes are therefore uncertain. Our results provide an exciting possibility by showing that inhibiting Jmjd2/Kdm 4 activity could be a feasible alternative treatment strategy for leukemia with high expression of IL3RA.

Binding of Il-3 to the receptor results in activation of receptor-associated JAKs and its downstream STAT pathway, which promotes both cell proliferation and survival. Although MLL rearrangements alone are sufficient to drive some forms of childhood leukemia, activating mutations downstream from Il3ra in, for instance, FLT3 and NRAS are often observed in combination with MLL rearrangements (Andersson et al. 2015). There is a possibility that mutations in FLT3 and NRAS could relieve the requirement for Jmjd2/Kdm4 during leukemic transformation, and it is important to address this in future studies.

Cheung et al. (2016) recently published the use of a chemical compound, SD70, to inhibit AML growth in vivo. Although the target specificity of SD70 is not known, the demonstration that it can inhibit the demethylation activity of JMJD2C/KDM4C in vitro (although with a low IC50 of $\sim 30 \mu \mathrm{M}$ ) (Jin et al. 2014) could be used as an argument to propose that this study provided some proof of concept for targeting the JMJD2/KDM4 demethylases in AML. In recent years, several mechanistic-based inhibitors toward the catalytic JmjC domain of the histone demethylases have been studied; however, so far, none of these have been shown to be selective for the JMJD2/KDM4 demethylases (Wang et al. 2013; Bavetsias et al. 2016; Westaway et al. 2016a,b). Because the JmjC demethylases can function as both tumor suppressors and oncogenes, it will be essential to develop subfamily-specific chemical inhibitors for therapeutic use. Based on the studies presented here, we hope that the available inhibitors will be refined so that, in a few years, we can test the feasibility of using JMJD2/KDM4-specific inhibitors for the treatment of AML.

\section{Material and methods}

Mice

Generation of $I m j d 2 a / b / c^{f / f} ;$ Rosa26::CreERT2 mice is described in Pedersen et al. (2016). B6.SJL mice were used as recipients in the transplantation experiments. Transplanted mice were inspected daily, and moribund mice were euthanized by cervical translocation. The emergence of AML was confirmed by the presence of Gr1/ Mac1-double-positive CD45.2 cells in the spleen. One representative FACS plot is shown in Figure 1D. All mouse work was approved by the Danish Animal Ethical Committee ("Dyreforsøgstilsynet") under license number 2012-15-2934-00224.

\section{BM transplantation}

c-Kit ${ }^{+}$BM cells from $J m j d 2 a / b / c^{f / f}{ }_{;}$Rosa26::CreERT2 and $I m j d 2 c^{f / f}$;Rosa26::CreERT2 mice were transduced with MSCV-MLL-AF9-neo. After 2 d, cells were plated in methylcellulose medium (Stem Cell Technologies, M3532) containing $50 \mathrm{ng} / \mathrm{mL} \mathrm{SCF}, 10 \mathrm{ng} / \mathrm{mL}$ IL-6, $10 \mathrm{ng} /$ $\mathrm{mL} \mathrm{IL-3,} \mathrm{and} \mathrm{neomycin.} \mathrm{Following} \mathrm{three} \mathrm{rounds} \mathrm{of} \mathrm{re-}$ plating, $5 \times 10^{4}$ preleukemic cells were transplanted into sublethally irradiated (600 rad) B6.SJL mice by tail vein injections.

\section{Virus production}

Phoenix-Ecotropic cells were transfected with MSCV vectors using the standard calcium phosphate transfection method. Forty-eight hours after removal of transfection mix, viral supernatants were harvested and centrifuged on Retronectin-coated plates that were subsequently used to transduce c-Kit ${ }^{+}$cells. The Gateway Entrycloneencoding murine Il3ra ORF was obtained from Genopedia and subcloned into MSCV using LR clonase.

\section{In vitro culture of $M L L$-AF9 transformed cells}

Liquid culture of MLL-AF9 and Hoxa9-Meis1 transformed cells was done in RPMI containing 20\% FBS and $10 \mathrm{ng} / \mathrm{mL}$ IL-3. Leukemic cell lines were derived by 
explanting cells from the BM of leukemic mice into RPMI containing $20 \%$ FBS and $10 \mathrm{ng} / \mathrm{mL}$ IL-3.

\section{EdU incorporation}

EdU incorporation assay was performed according to the manufacturer's protocol using Click-It EdU assay (Thermo Fisher).

\section{RNA and gene expression analyses}

RNA was purified using the RNeasy kit (Qiagen) and reverse-transcribed using TaqMan reverse transcription reagents (Applied Biosystems) according to the manufacturer's instructions. In RT-qPCR experiments, expression values were normalized to the housekeeping gene Rplp0. All qPCR reactions were performed on a Roche LightCycler 480 II using SYBR Green master mix. For microarray analysis, RNA from three independently derived MA92 abc leukemic cell lines was isolated after $96 \mathrm{~h}$ of treatment with $500 \mathrm{nM}$ OHT or control. Samples were labeled and hybridized to Agilent SurePrint G3 mouse GE 8x60K arrays (Agilent, G4852A) according to the manufacturer's instructions using LowInput QuickAmp One-Color labeling kit (Agilent, 5190-2305), One-Color RNA Spike-In kit (Agilent, 5188-5282), and gene expression hybridization kit (Agilent, 5188-5242). Array probe annotation was downloaded from the Agilent Web site and subsequently converted to an $\mathrm{R}$ package using AnnotationForge. In $\mathrm{R}$, we performed background correction ("normexp," offset =75), normalization ("cyclicloess"), filtering, and statistical analysis using the limma package. Test $P$-values were converted to FDR values to adjust for multiple testing.

\section{Antibodies}

The specificity of $\mathrm{H} 3 \mathrm{~K} 9 \mathrm{me} 3$ antibodies was tested in ELISA experiments with a histone peptide library (Pedersen et al. 2014). Polyclonal antibodies against Jmjd2b and Jmjd2c have been described previously (Pedersen et al. 2014). The following commercially available antibodies were used: anti-Jmjd2a (Cell Signaling Technology, 5328), anti- $\beta$ actin (Abcam, ab6276), anti-H3K9me3 (Abcam, ab8898), anti-H3 (Abcam, ab1791), anti-mouse CD11b (Biolegend, clone MI78), and anti-mouse Ly-6g/ Ly-6c (Biolegend, RB6-8C5).

\section{Western blot analysis}

Protein lysates and Western blot analysis were done using standard protocols.

\section{GSEA}

GSEA (Subramanian et al. 2005) was performed with GSEA version 2.2.1 software obtained from the Broad Institute Web site. The PID_IL3_PATHWAY gene set was obtained from the Molecular Signature Database, and the gene set induced by IL-3 treatment of human
AML samples (sustained response) was described in Sadras et al. (2014).

\section{ChIP and ChIP-seq}

ChIP experiments were performed as described in KleineKohlbrecher et al. (2010), and ChIP-seq libraries were made using NEBNext Ultra II DNA library preparation kit for Illumina according to the manufacturer's protocol. Reads were mapped to the mouse genome (mm10 assembly) using Bowtie for Illumina (Galaxy tool version 1.1.2) (Langmead et al. 2009). Mapped reads were filtered for PCR duplicates using RmDup (version 0.1.19). Peak calling and visualization of tracks were done using EaSeq (http://www.easeq.net) (Lerdrup et al. 2016), and heat maps were made using seqMINER (Ye et al. 2011). ChIPseq tracks of $\mathrm{H} 3 \mathrm{~K} 4 \mathrm{me} 3$ in mouse leukemia stem cells were visualized using EaSeq with data from GSE29130 (Bernt et al. 2011). Chromosomal positions were annotated according to the RefSeq database (mm10; January 19, 2015) using the University of California at Santa Cruz refFlat tables. In our annotation, a TSS region is defined as $\pm 1 \mathrm{~kb}$ relative to the TSS. For density plots, we generated bigwig files allowing only one read per chromosomal position, eliminating potential spurious spikes. Each remaining read was extended from its $5^{\prime}$ end to a total length of 250 bases. Each bigwig file was also scaled to TPM /tags per million) based on the number of unique read positions. For density plots, the regions of $\pm 5 \mathrm{~kb}$ for all annotated TSSs were divided into 100 equal-sized bins.

\section{Primer sequences}

For RT-qPCR, the primer sequences used were Jmjd2a_F (AAGAAAGCCATGACCGTTCGTG), Jmjd2a_R(AAAT TCACTGTATCGCGGGGTG), Jmjd2b_F (GATCATGA CTTTCCGCCCCA), Jmjd2b_R (TCATCATACGTCTG CCGTGG), Jmjd2c_F (GCCAGATAGATACCAGATTT G), Jmjd2c_R (GGCAGGGTTTGCACCCTCTTC), Il3ra_F (CTGTGAAGACAGCCTTGGTG), Il3ra_R (GCGGTAG AGCAGCGACTTC), Rp0lp_F(TTCATTGTGGGAGCAGAC), and Rp0lp_R (CAGCAGTTTCTCCAGAGC).

For ChIP-qPCR, the primer sequences used were Il3ra_TSS_F (ACTTGCCAGCATCCTCCA) and Il3ra_ TSS_R (AGTGCAGATGACAAGGCAGA).

\section{Accession codes}

ChIP-seq and microarray data have been deposited in the Gene Expression Omnibus (GEO) database with accession number GSE81300.

\section{Acknowledgments}

We thank the members of the Helin laboratory for discussion, technical advice, and support, and in particular Lotte Holck and Fengqin Jia for expert technical assistance. We thank Kirsten Grønbæk and Bo T. Porse for critical comments on the manuscript. We thank Mads Lerdrup for discussion and technical advice regarding the use of EaSeq. S.M.K. was supported by a post- 
doctoral fellowship from the Netherlands Organization for Scientific Research (NWO). This work was supported by the Danish Cancer Society, the Danish National Research Foundation (DNRF 82), the Lundbeck Foundation, and a center grant from the Novo Nordisk Foundation (The Novo Nordisk Foundation Section for Stem Cell Biology in Human Disease).

\section{References}

Andersson AK, Ma J, Wang J, Chen X, Gedman AL, Dang J, Nakitandwe J, Holmfeldt L, Parker M, Easton J, et al. 2015. The landscape of somatic mutations in infant MLL-rearranged acute lymphoblastic leukemias. Nat Genet 47: 330-337.

Bavetsias V, Lanigan RM, Ruda GF, Atrash B, McLaughlin MG, Tumber A, Mok NY, Le Bihan YV, Dempster S, Boxall KJ, et al. 2016. 8-substituted pyrido[3,4-d]pyrimidin-4(3H)-one derivatives as potent, cell permeable, KDM4 (JMJD2) and KDM5 (JARID1) histone lysine demethylase inhibitors. J Med Chem 59: 1388-1409.

Bernt KM, Armstrong SA. 2011. A role for DOT1L in MLL-rearranged leukemias. Epigenomics 3: 667-670.

Bernt KM, Zhu N, Sinha AU, Vempati S, Faber J, Krivtsov AV, Feng Z, Punt N, Daigle A, Bullinger L, et al. 2011. MLL-rearranged leukemia is dependent on aberrant H3K79 methylation by DOT1L. Cancer Cell 20: 66-78.

Berry WL, Janknecht R. 2013. KDM4/JMJD2 histone demethylases: epigenetic regulators in cancer cells. Cancer Res 73: 2936-2942.

Broughton SE, Dhagat U, Hercus TR, Nero TL, Grimbaldeston MA, Bonder CS, Lopez AF, Parker MW. 2012. The GM-CSF/ IL-3/IL-5 cytokine receptor family: from ligand recognition to initiation of signaling. Immunol Rev 250: 277-302.

Cai SF, Chen CW, Armstrong SA. 2015. Drugging chromatin in cancer: recent advances and novel approaches. Mol Cell 60: 561-570.

Chen CW, Armstrong SA. 2015. Targeting DOT1L and HOX gene expression in MLL-rearranged leukemia and beyond. Exp Hematol 43: 673-684.

Cheung N, Fung TK, Zeisig BB, Holmes K, Rane JK, Mowen KA, Finn MG, Lenhard B, Chan LC, So CW. 2016. Targeting aberrant epigenetic networks mediated by PRMT1 and KDM4C in acute myeloid leukemia. Cancer Cell 29: 32-48.

Cloos PA, Christensen J, Agger K, Maiolica A, Rappsilber J, Antal T, Hansen KH, Helin K. 2006. The putative oncogene GASC1 demethylates tri- and dimethylated lysine 9 on histone $\mathrm{H} 3$. Nature 442: 307-311.

de Boer J, Walf-Vorderwulbecke V, Williams O. 2013. In focus: MLL-rearranged leukemia. Leukemia 27: 1224-1228.

Fodor BD, Kubicek S, Yonezawa M, O'Sullivan RJ, Sengupta R, Perez-Burgos L, Opravil S, Mechtler K, Schotta G, Jenuwein T. 2006. Jmjd2b antagonizes H3K9 trimethylation at pericentric heterochromatin in mammalian cells. Genes Dev 20: 1557-1562.

Hojfeldt JW, Agger K, Helin K. 2013. Histone lysine demethylases as targets for anticancer therapy. Nat Rev Drug Discov 12: 917-930.

Iwamori N, Zhao M, Meistrich ML, Matzuk MM. 2011. The testis-enriched histone demethylase, KDM4D, regulates methylation of histone $\mathrm{H} 3$ lysine 9 during spermatogenesis in the mouse but is dispensable for fertility. Biol Reprod 84: 1225-1234.

Jin L, Lee EM, Ramshaw HS, Busfield SJ, Peoppl AG, Wilkinson L, Guthridge MA, Thomas D, Barry EF, Boyd A, et al. 2009. Monoclonal antibody-mediated targeting of CD123, IL-3 re- ceptor a chain, eliminates human acute myeloid leukemic stem cells. Cell Stem Cell 5: 31-42.

Jin C, Yang L, Xie M, Lin C, Merkurjev D, Yang JC, Tanasa B, Oh S, Zhang J, Ohgi KA, et al. 2014. Chem-seq permits identification of genomic targets of drugs against androgen receptor regulation selected by functional phenotypic screens. Proc Natl Acad Sci 111: 9235-9240.

Jordan CT, Upchurch D, Szilvassy SJ, Guzman ML, Howard DS, Pettigrew AL, Meyerrose T, Rossi R, Grimes B, Rizzieri DA, et al. 2000. The interleukin-3 receptor $\alpha$ chain is a unique marker for human acute myelogenous leukemia stem cells. Leukemia 14: 1777-1784.

Kawazu M, Saso K, Tong KI, McQuire T, Goto K, Son DO, Wakeham A, Miyagishi M, Mak TW, Okada H. 2011. Histone demethylase JMJD2B functions as a co-factor of estrogen receptor in breast cancer proliferation and mammary gland development. PLoS One 6: e17830.

Kleine-Kohlbrecher D, Christensen J, Vandamme J, Abarrategui I, Bak M, Tommerup N, Shi X, Gozani O, Rappsilber J, Salcini $\mathrm{AE}$, et al. 2010. A functional link between the histone demethylase PHF8 and the transcription factor ZNF711 in X-linked mental retardation. Mol Cell 38: 165-178.

Klose RJ, Yamane K, Bae Y, Zhang D, Erdjument-Bromage H, Tempst P, Wong J, Zhang Y. 2006. The transcriptional repressor JHDM3A demethylates trimethyl histone $\mathrm{H} 3$ lysine 9 and lysine 36. Nature 442: 312-316.

Kooistra SM, Helin K. 2012. Molecular mechanisms and potential functions of histone demethylases. Nat Rev Mol Cell Biol 13: 297-311.

Kouzarides T. 2007. Chromatin modifications and their function. Cell 128: 693-705.

Krivtsov AV, Twomey D, Feng Z, Stubbs MC, Wang Y, Faber J, Levine JE, Wang J, Hahn WC, Gilliland DG, et al. 2006. Transformation from committed progenitor to leukaemia stem cell initiated by MLL-AF9. Nature 442: 818-822.

Kroon E, Krosl J, Thorsteinsdottir U, Baban S, Buchberg AM, Sauvageau G. 1998. Hoxa9 transforms primary bone marrow cells through specific collaboration with Meisla but not Pbxlb. EMBO I 17: 3714-3725.

Langmead B, Trapnell C, Pop M, Salzberg SL. 2009. Ultrafast and memory-efficient alignment of short DNA sequences to the human genome. Genome Biol 10: R25.

Lerdrup M, Johansen JV, Agrawal-Singh S, Hansen K. 2016. An interactive environment for agile analysis and visualization of ChIP-sequencing data. Nat Struct Mol Biol 23: 349-357.

Liu K, Zhu M, Huang Y, Wei S, Xie J, Xiao Y. 2015. CD123 and its potential clinical application in leukemias. Life Sci 122: 59-64.

Luo W, Chang R, Zhong J, Pandey A, Semenza GL. 2012. Histone demethylase JMJD2C is a coactivator for hypoxia-inducible factor 1 that is required for breast cancer progression. Proc Natl Acad Sci 109: E3367-E3376.

Muntean AG, Hess JL. 2012. The pathogenesis of mixed-lineage leukemia. Annu Rev Pathol 7: 283-301.

Nishinakamura R, Nakayama N, Hirabayashi Y, Inoue T, Aud D, McNeil T, Azuma S, Yoshida S, Toyoda Y, Arai K, et al. 1995. Mice deficient for the IL-3/GM-CSF/IL-5 $\beta$ c receptor exhibit lung pathology and impaired immune response, while $\beta$ IL3 receptor-deficient mice are normal. Immunity 2: 211-222.

Pedersen MT, Agger K, Laugesen A, Johansen JV, Cloos PA, Christensen J, Helin K. 2014. The demethylase JMJD2C localizes to H3K4me3-positive transcription start sites and is dispensable for embryonic development. Mol Cell Biol 34: 1031-1045. 
Pedersen MT, Kooistra SM, Radzisheuskaya A, Laugesen A, Johansen JV, Hayward DG, Nilsson J, Agger K, Helin K. 2016. Continual removal of H3K9 promoter methylation by Jmjd2 demethylases is vital for ESC self-renewal and early development. $E M B O J$ (in press).

Rao RC, Dou Y. 2015. Hijacked in cancer: the KMT2 (MLL) family of methyltransferases. Nat Rev Cancer 15: 334-346.

Sadras T, Perugini M, Kok CH, Iarossi DG, Heatley SL, Brumatti G, Samuel MS, To LB, Lewis ID, Lopez AF, et al. 2014. Interleukin-3-mediated regulation of $\beta$-catenin in myeloid transformation and acute myeloid leukemia. J Leukoc Biol 96: 83-91.

Shi L, Sun L, Li Q, Liang J, Yu W, Yi X, Yang X, Li Y, Han X, Zhang $\mathrm{Y}$, et al. 2011. Histone demethylase JMJD2B coordinates H3K4/H3K9 methylation and promotes hormonally responsive breast carcinogenesis. Proc Natl Acad Sci 108: 75417546.

Somervaille TC, Cleary ML. 2006. Identification and characterization of leukemia stem cells in murine MLL-AF9 acute myeloid leukemia. Cancer Cell 10: 257-268.

Subramanian A, Tamayo P, Mootha VK, Mukherjee S, Ebert BL, Gillette MA, Paulovich A, Pomeroy SL, Golub TR, Lander ES, et al. 2005. Gene set enrichment analysis: a knowledgebased approach for interpreting genome-wide expression profiles. Proc Natl Acad Sci 102: 15545-15550.

Testa U, Riccioni R, Militi S, Coccia E, Stellacci E, Samoggia P, Latagliata R, Mariani G, Rossini A, Battistini A, et al. 2002. Elevated expression of IL-3Ra in acute myelogenous leukemia is associated with enhanced blast proliferation, increased cellularity, and poor prognosis. Blood 100: 2980-2988.
Testa U, Pelosi E, Frankel A. 2014. CD 123 is a membrane biomarker and a therapeutic target in hematologic malignancies. Biomark Res 2: 4.

Wang Y, Krivtsov AV, Sinha AU, North TE, Goessling W, Feng Z, Zon LI, Armstrong SA. 2010. The Wnt $/ \beta$-catenin pathway is required for the development of leukemia stem cells in AML. Science 327: 1650-1653.

Wang L, Chang J, Varghese D, Dellinger M, Kumar S, Best AM, Ruiz J, Bruick R, Pena-Llopis S, Xu J, et al. 2013. A small molecule modulates Jumonji histone demethylase activity and selectively inhibits cancer growth. Nat Commun 4: 2035.

Westaway SM, Preston AG, Barker MD, Brown F, Brown JA, Campbell M, Chung CW, Diallo H, Douault C, Drewes G, et al. 2016a. Cell penetrant inhibitors of the KDM4 and KDM5 families of histone lysine demethylases. 1. 3-amino4-pyridine carboxylate derivatives. I Med Chem 25: 13571369.

Westaway SM, Preston AG, Barker MD, Brown F, Brown JA, Campbell M, Chung CW, Drewes G, Eagle R, Garton N, et al. 2016b. Cell penetrant inhibitors of the KDM4 and KDM5 families of histone lysine demethylases. 2. Pyrido [3,4-d]pyrimidin-4(3H)-one derivatives. I Med Chem 25: $1370-1387$.

Whetstine JR, Nottke A, Lan F, Huarte M, Smolikov S, Chen Z, Spooner E, Li E, Zhang G, Colaiacovo M, et al. 2006. Reversal of histone lysine trimethylation by the JMJD2 family of histone demethylases. Cell 125: 467-481.

Ye T, Krebs AR, Choukrallah MA, Keime C, Plewniak F, Davidson I, Tora L. 2011. seqMINER: an integrated ChIP-seq data interpretation platform. Nucleic Acids Res 39: e35. 


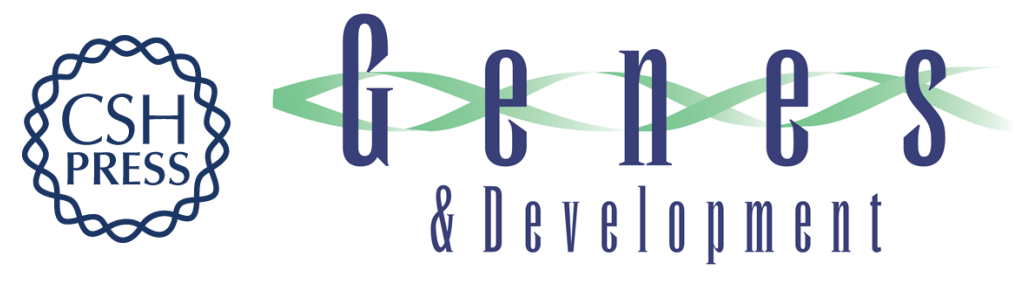

\section{$\mathrm{Jmjd2} / \mathrm{Kdm} 4$ demethylases are required for expression of //3ra and survival of acute myeloid leukemia cells}

Karl Agger, Satoru Miyagi, Marianne Terndrup Pedersen, et al.

Genes Dev. 2016, 30: originally published online June 2, 2016

Access the most recent version at doi:10.1101/gad.280495.116

\section{Supplemental http://genesdev.cshlp.org/content/suppl/2016/06/02/gad.280495.116.DC1 \\ Material}

References This article cites 44 articles, 10 of which can be accessed free at:

http://genesdev.cshlp.org/content/30/11/1278.full.html\#ref-list-1

Creative This article is distributed exclusively by Cold Spring Harbor Laboratory Press for the first

Commons six months after the full-issue publication date (see

License http://genesdev.cshlp.org/site/misc/terms.xhtml). After six months, it is available under a Creative Commons License (Attribution-NonCommercial 4.0 International), as described at http://creativecommons.org/licenses/by-nc/4.0/.

Email Alerting Receive free email alerts when new articles cite this article - sign up in the box at the top Service right corner of the article or click here.

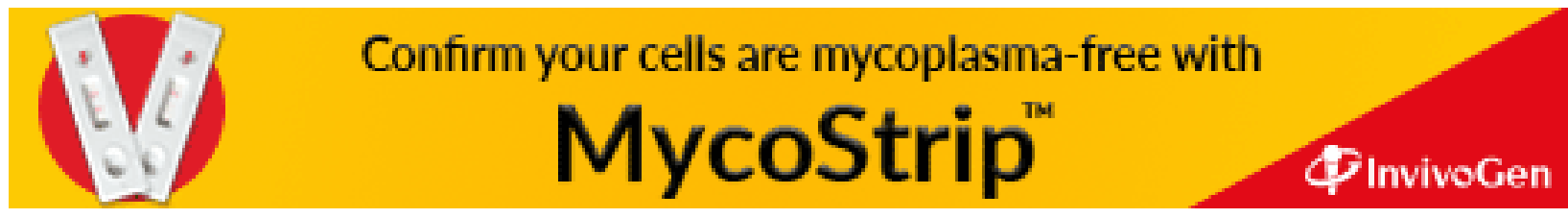

